\title{
Is the ejection of the corona a general phenomenon in microquasars?
}

\author{
Jérôme Rodriguez* \\ CEA Saclay, IRFU/Service D'Astrophysique, UMR AIM, France \\ E-mail: jrodriguez@cea.fr

\section{Lionel Prat} \\ CEA Saclay, IRFU/Service D'Astrophysique, UMR AIM, France \\ E-mail: lionel.prat@cea.fr
}

\begin{abstract}
We study the evolution of some microquasars during their outbursts as observed with the X-ray telescopes RXTE and INTEGRAL. We focus on the interplay between the accretion disc, and the medium responsible for the production of the hard X-rays (the so-called corona). By comparing the behaviour of two sources (XTE J1550-564 and GRS 1915+105) at X-ray energies and radio wavelengths, we propose a scenario in which the discrete ejections are triggered in coincidence with soft X-ray peaks during the outburst. We also suggest, in those two sources, that the ejected material is the corona that is seen to disappear in coincidence with the X-ray maxima. We then turn to two other sources, XTE J1748-248, and XTE J1859+226, and study whether the same conclusions can be drawn from the existing multi-wavelength (radio+X-ray) data.
\end{abstract}

7th INTEGRAL Workshop

September 8-11 2008

Copenhagen, Denmark

\footnotetext{
*Speaker.
} 


\section{Introduction}

Up to now, about 20 black hole (BH) X-ray transients have been observed, along with 20 more candidates black-hole binaries [9]. The huge archival X-ray observations available allowed different spectral states to be identified. These depend on both the spectral and temporal characteristics of the source [7,9]. Radio observations have shown that these objects, through their outbursts, usually show the presence of radio jets. This behaviour, also seen in quasars that are thought to host super-massive BH, led, by analogy, to call them 'microquasars' [11]. Since then, systematic quasi-simultaneous multi-wavelength observations (i.e. combining, at least, radio and X-ray observations), have permitted researchers to identify a common pattern for the evolution of microquasars through their outbursts, referred to as the "Q-shape" given the shape it draws on a hardness-intensity diagram (HID, Fig. 1, [5]). This approach has the advantage of being model independent. It is, however, interpreted in terms of a generally admitted model, involving an accretion disc, responsible for the emission of soft X-rays, a so-called "corona" responsible for the hard $\mathrm{X}$-ray tail, and a jet responsible for the radio and part of the infrared emissions. Note that there is no consensus as to whether the corona and the jet are different media, and some models predict that the jet could be responsible of (part of) the hard X-ray emission [10].

An outburst starts in the "low" hard state (LHS). The spectrum is hard, dominated by the coronal emission (inverse Comptonization on a population of thermal electrons). The X-rays show a high level of variability and some quasi-periodic oscillations (QPOs), while a compact jet is seen in the radio bands. In this state the radio and soft X-ray fluxes are known to be correlated [3]. During the outburst, the source may reach the soft state (SS), in which the hard tail is almost completely absent, and the X-rays are entirely dominated by the disc emission. There is no jet in this state. The transition from LHS to SS occurs through different intermediate states, and through the socalled jet line. In this top horizontal branch of the HID (Fig. 1), the compact jet is quenched, while the crossing of the jet line is accompanied by discrete ejections of material [5]. At the end of the outburst, the source returns to quiescence after it has transited again the the LHS. A key to fully understand the physics of these states may reside in deeper and more systematic studies of the state transitions, especially with physical diagnostics.

In this paper we initiate a systematic X-ray study of microquasars in dates preceding radio ejections. This follows earlier works by [14] and [16, 17] on, respectively, XTE J1550-564 and GRS 1915+105, who showed in particular similar evolution at times preceding ejections.

We start this paper by summarising the results obtained in XTE J1550-564 and GRS 1915+105 in Section 2 and 3, and then turn to two other sources, XTE J1748-248, and XTE J1859+226, for which quasi-simultaneous radio and X-ray observations exist. We discuss our results in the last part of the paper.

\section{XTE J1550-564: the beginning of the story?}

The 2000 outburst of XTE J1550-564 ( 70 day) was monitored in a multi-wavelength approach. Mainly, this included X-ray energies with RXTE, and radio and infrared wavelengths from ground based facilities. [4] reported the detection of radio emission soon after the $2-12 \mathrm{keV}$ maximum of the outburst. This radio emission was interpreted as due to a discrete ejection of material, 
Figure 1: Typical schematic evolution of a microquasar through an outburst. The central panel shows the hardness-intensity diagram obtained from the X-ray observations, with, on each sides, the corresponding behaviour of the accretion disc and the jet. The outer panels show the corresponding behaviour in the Fourier domain. Adapted from $[5,9,7]$.
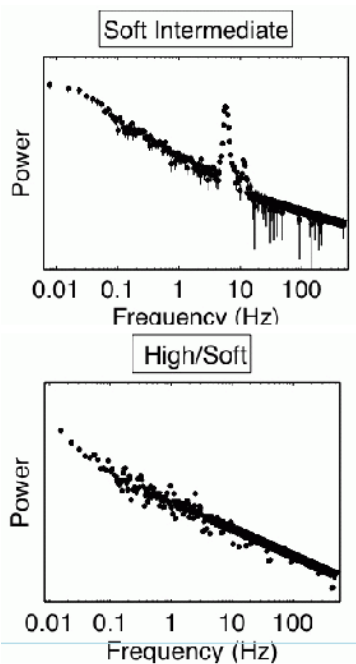

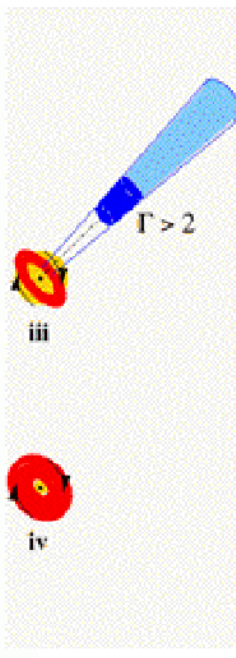

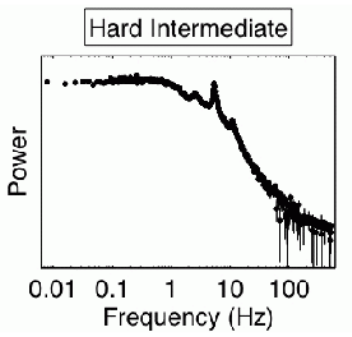

| Low/Hard |

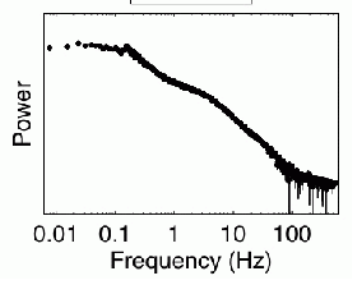

the precise date of which could not be determined. $[14,15]$ presented a precise spectral and timing analysis of all RXTE observations of this outburst. The energy spectra were fitted with the common model involving emission from an accretion disc, a power law tail (either with a high energy cut-off or not), an iron line and an iron edge around $8 \mathrm{keV}$. The evolution of the main spectral parameters, and the identification of the spectral states (according to the classification of [9] are reported in Fig. 2. The cut-off power law is commonly thought to represent a process of thermal Comptonization, that occurs in the corona. The absence of a cut-off is usually taken as evidence that the corona is no more thermalised (i.e. the electrons no longer have a Maxwellian distribution of their velocities). During the 2000 outburst of XTE J1550-564, the cut-off power law disappeared about 2 days after the first transition, on MJD 51662, and reappeared after the second transition, when the source went back to the LHS, on MJD 51682 (Fig. 2). Soon after the X-ray maximum (after MJD 51662), while the disc flux remained approximately constant over few days, the power law flux decreased significantly. At the same time the power law photon index remained roughly constant, which indicates that the decrease of the power law flux is not due to a pivoting of the X-ray spectrum but traces the fact that something drastic changed in the corona. The large decrease of the coronal flux may indicate that part of the corona disappeared, ie, that it was either accreted by the $\mathrm{BH}$, or ejected. The detection of a discrete ejecta soon after the X-ray peak makes it tempting to consider that the Compton medium may have been blown away.

\section{GRS 1915+105: towards a generalisation of the ejection of the corona}

GRS 1915+105 has been widely observed at all wavelengths since its discovery in 1992. First connection between accretion and ejection phenomena were reported by [12], when these authors remarked that during in a particular observation, X-ray episodes of low and spectrally hard state 
Figure 2: Left : Evolution of the main spectral parameters of XTE J1550-564 during the 2000 outburst. Right : Evolution of the $2-50 \mathrm{keV}$ disc flux vs. the $2-50 \mathrm{keV}$ power law flux over the period of outburst. The state prescription is that of [9]. Both figures adapted from [14].
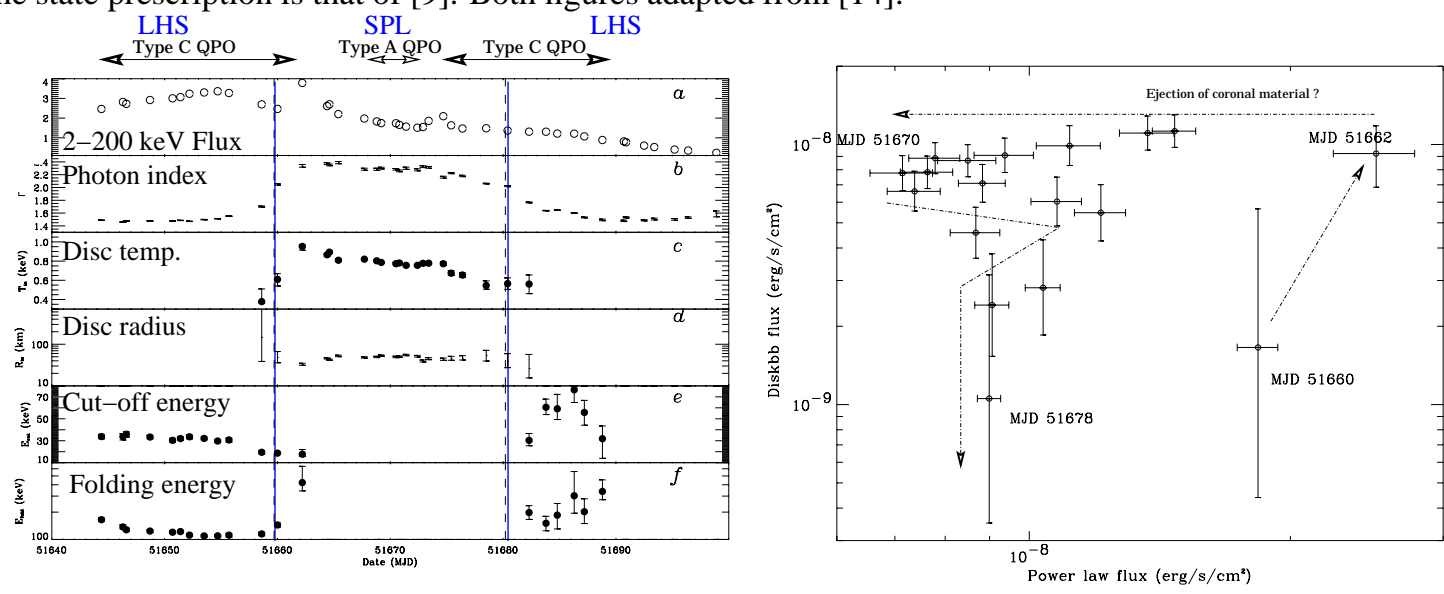

Figure 3: Zoom on a portion of the INTEGRAL and Ryle observations of GRS $1915+105$ showing three particular cycles, each followed by a radio flare. The 3 observations belong to classes $v, \lambda$, and $\beta$. From [17].
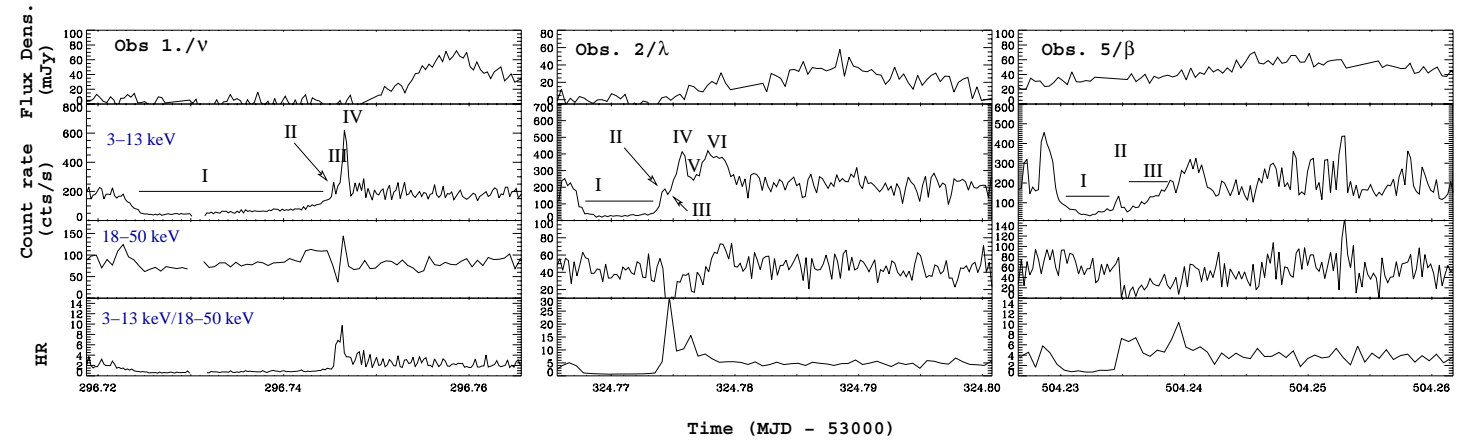

terminated by a sudden X-ray spike marking the transition to a softer state (hereafter "cycle"), were followed by flares, seen in infrared first and later in radio. These were interpreted as due to discrete ejection of plasmoids.

We (PIs Hannikainen and Rodriguez) are conducting a monitoring campaign of GRS 1915+105 with INTEGRAL since 2003. This campaign is, as much as possible, conducted in coordination with radio observations. Over the period 2004-2005, we were lucky enough to catch the source in several of its variability states (Fig. 3) as defined by [1]. So far, amongst all classes showing cycles (the so-called $\alpha, \beta, v, \lambda, \theta$ ), only the cycles of class $\lambda$ had not been seen to be associated to radio flares, and thus to ejections [8]. The observation of an ejection during a $\lambda$ observation was the first ever reported. It has a great importance as it allowed us to generalise the following: in GRS $1915+105$, discrete plasmoid ejections always occurs as a response to an X-ray cycle composed of a spectrally hard X-ray dip (longer than 100s) ended by a sudden X-ray spike marking the return to a spectrally soft state (Fig. 3, [16]). We indeed showed that, from both spectral and temporal points of view, theses classes were qualitatively similar. They all show the presence of 
Figure 4: Spectral evolution of GRS 1915+105 during the cycle of class $v$. The fit-model consist of disc black body and Comptonised emission both absorbed.
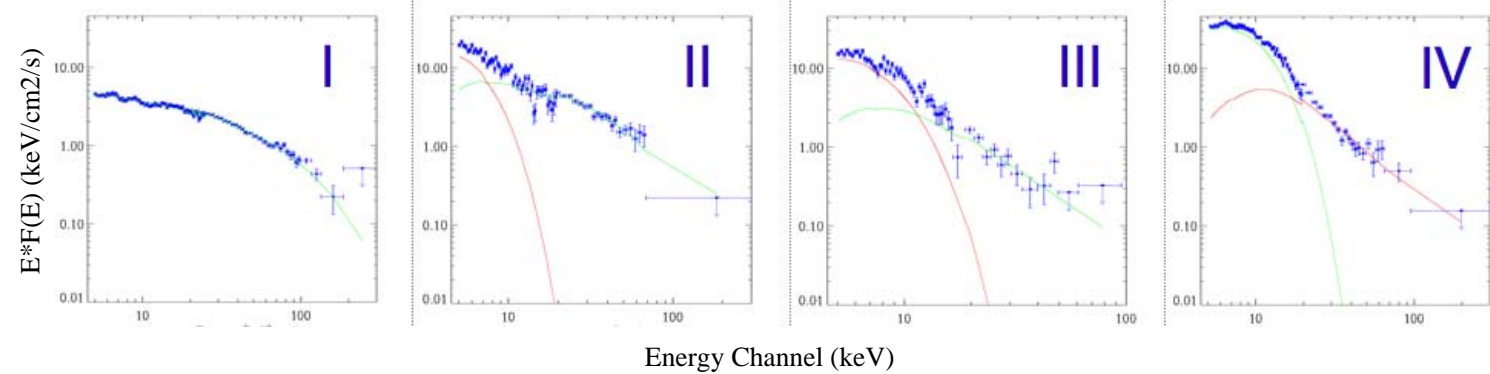

low frequency QPOs of variable frequency during the X-ray dip. The QPO disappears at the spike. The delay between the X-ray spike and the radio peak is similar (about 0.3 hours). The spectral evolution during the cycle is similar (although the exact spectral parameters may have different absolute values). There exists a possible correlation between the duration of the X-ray dip and the amplitude of the radio flare, which may strongly link what happens in the disc-corona system prior to the ejection [16].

The generalisation of the accretion-ejection links in GRS 1915+105 was shown in two different ways, a model-independent one (i.e. based on light curves and hardness ratios [16], not shown here), and through fits to the spectra ([17], Fig. 4). The cycles were divided into different intervals based on the hardness ratio of the source. All spectra were fitted with a model accounting for the emission of the disc (ezdiskbb) and that of the corona (comptt), both modified by absorption. In all cases, the transition from interval II to III manifests by a drastic change in the coronal properties. First the corona seems to completely change from thermalised to non-thermalised. Then the flux of the corona shrinks while that of the disc remains constant. Again, all the points mentioned above, and the spectral evolution of the source through the cycle, leads us to suggest that (part of) the corona is ejected $[16,17]$.

\section{What happens in other sources : the cases of XTE J1859+226 and XTE J1748-288}

The common behaviour of XTE J1550-564 and GRS 1915+105, at least regarding the origin of the ejected material, led us to search for the same evolutionary pattern in other sources. Here we present the results obtained for two more microquasars.

\subsection{Evolution of the fluxes: a different picture?}

XTE J1748-288 is another transient source that was discovered by RXTE in 1998. [13] report the spectral analysis of the RXTE data over the outburst. Although they analyse the data with phenomenological models, they remark that the $15-30 \mathrm{keV}$ flux decreased more rapidly than the soft X-ray flux after the peak of the outburst. A multiwavelength light curve of this 1998 outburst, with the Green Bank Interferometer in radio, RXTE at X-ray energies, and CGRO/BATSE in the hard X-rays, is shown in the left panel of Fig. 5. 
Figure 5: Multiwavelength evolution of XTE J1748-288 (Left) over its 1998 outburst, and XTE J1859+226 (Right) over its 1999 outburst. From top to bottom, the panels show the radio light curves seen with the GBI, the soft (RXTE) and hard (BATSE) X-rays, intermediate X-rays as seen with RXTE/PCA, and the hardness ratio between two X-ray bands.
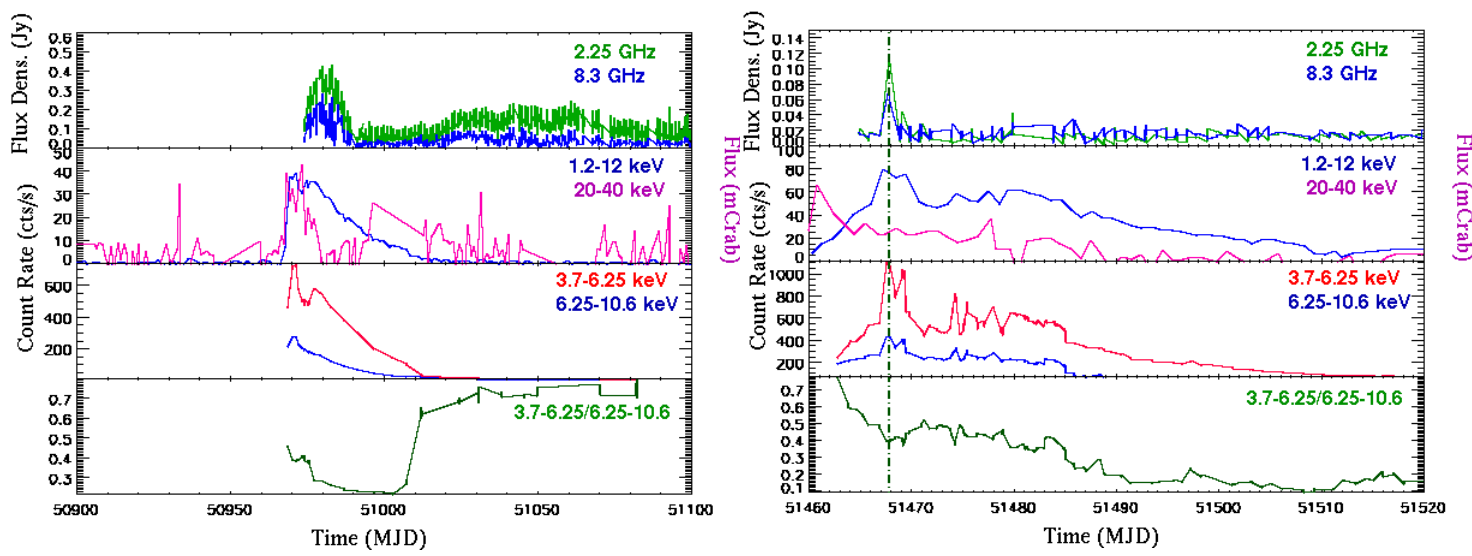

XTE J1859+226 was also discovered by RXTE in 1999 [Woods et al.], when entering an Xray outburst. The multiwavelength light curve is shown in Fig 5. A deep radio analysis is presented by [2] who remark that the source showed multiple ejection events during the outburst. The multiwavelength light curve is shown in the right panel of Fig 5. Similarly to XTE J1550-564 and GRS $1915+105$ (although on a much longer time scale), the same kind of qualitative evolution can be seen in these two sources. The outburst starts at hard X-rays. The soft X-ray peak is followed by a radio flare, indicative of an ejection of material.

We reduced the RXTE (PCA+HEXTE) data of all the observations from the beginning of the outburst of the two sources until the peak of the radio emission. The data reduction was performed in a very standard way (in particular concerning the time filtering, production of light curves and spectra). See, for example, $[14,17]$ for the details of the RXTE data reduction which are identical. The resulting spectra were fitted in XSPEC with a model consisting of a disc component (diskbb) and Comptonization (comptt) to account for the hard X-ray emission. All these components were convolved by low energy absorption. The choice of this particular model (as opposed to a simple power law) is dictated by the fact that the interplay between the disc and the corona is more physical at energies $<3 \mathrm{kT}_{\text {disc }}$. In particular the model suffers less of the mixing of a high level power law in the region where the disc emits at maximum. Therefore, the fluxes of the two components are more realistic. In all cases the temperature of the seed photons for Comptonization was equalised to that of the disc. For both sources we therefore obtained all spectral parameters: disc temperature, disc inner radius, temperature of the Comptonising electrons, optical depth of the Compton component, and the unabsorbed fluxes of the two spectral components. The detailed spectral results will be presented in a future paper (Rodriguez \& Prat, in prep.).

Here we focus on the evolution of the fluxes of the two spectral components vs. time, and that of the $2-50 \mathrm{keV}$ disc flux one vs. the $2-50 \mathrm{keV}$ comptonised flux, which are represented in Fig. 6 . In both cases, we also represented significant radio events (beginning and/or maxima) with vertical 
Figure 6: Evolution of the fluxes of the different spectral components (disc in green and Comptonised component in blue) vs time (top panel). Evolution of the 2-50 keV disc flux vs. the 2-50 keV Comptonised flux (bottom panel). The left side of the figure corresponds to the data of XTE J1748-288, while the right side corresponds to XTE J1859+226. The vertical lines represent the dates of the first radio detection and of the radio maximum for XTE J1748-288, and that of the first detection of an optically thin radio ejection in XTE J1859+226.
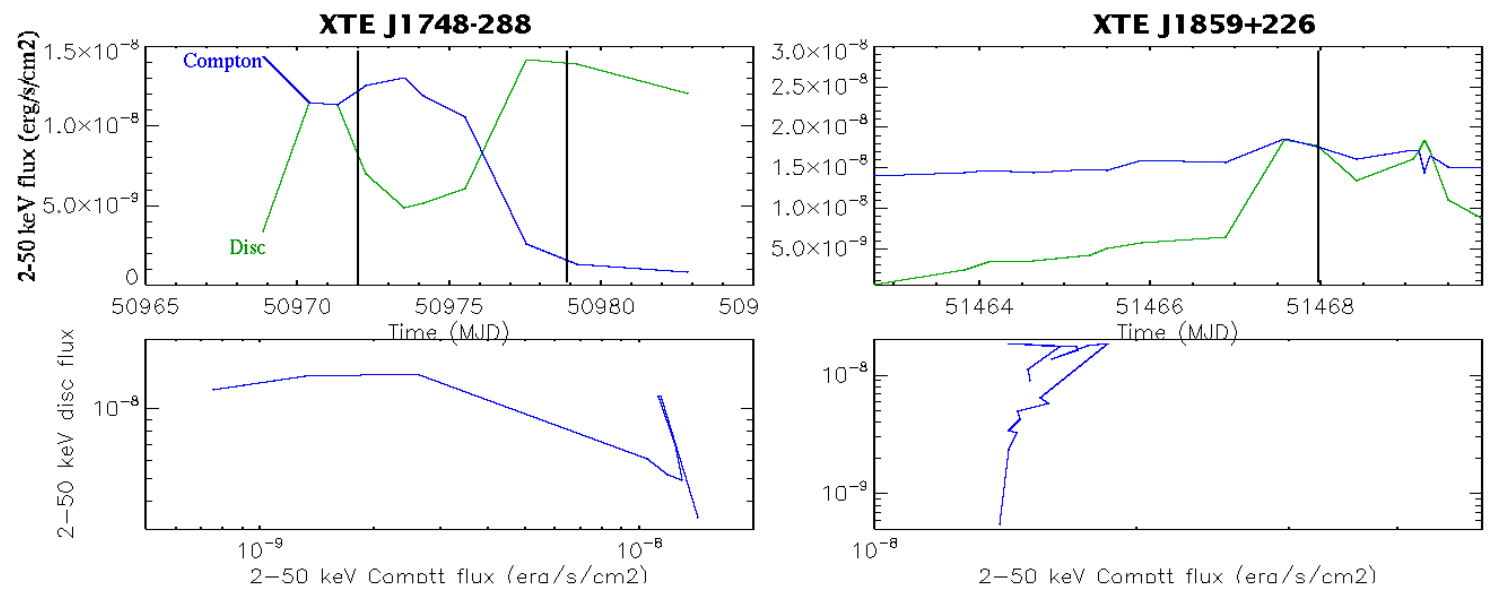

lines. In XTE J1748-288 a significant decrease of the Comptonised flux is seen prior to the radio peak, and this could be indicative, as in the previous two sources, of ejection of coronal material. The date of the first radio detection ([6]), however, occured before the decrease was initiated. It even occured after a slight decrease is seen in the flux of the accretion disc (Fig. 6). This therefore seems to contradict the picture we drew in the cases of XTE J1550-564 and GRS 1915+105. The huge decrease in coronal flux, however, may still indicate that a significant amount of coronal material feeds the jet.

The case of XTE J1859+226 is apparently completely at odds with this interpretation. The flux of the Comptonised component remains roughly constant over the period of interest (Fig. 6). This source is even more puzzling as the disc flux also shows an opposite behaviour to what would (naively) be expected. Indeed, it increases before the radio flare.

\subsection{Do we look at the right parameters?}

We look at the spectral components of the accretion flow and search if any of the two undergoes significant evolution. In XTE J1550-564 and GRS 1915+105 the drop in the flux of the Comptonised component led us to conclude that the ejected matter detected soon after this decrease was made of coronal material. The picture may, however, not be so simple in all sources, as Comptonization and transitions from a thermalised corona to a non thermalised one are not trivial phenomena. The evolution of fluxes may not be the appropriate parameter to look at. Indeed, one may think of a system in which the amount of seed photons increases such that even with a shrink of Comptonising electrons, the hard X-ray flux could appear as not varying much. In fact, the right parameter to characterise Comptonization is the so-called $y$ parameter ([18]) that somehow traduces the efficiency of Comptonization. $y$ can be expressed in terms of physical quantities characterising the corona, since $y \propto k T_{e} \times \max \left(\tau, \tau^{2}\right)$, with $k T_{e}$ the temperature of the coronal electrons, and $\tau$ the 
Figure 7: Evolution of the $y$ and $\tau$ parameters during the outburst of XTE J1748-288 (left) and XTE J1859+226 (right). In both cases, the top panels shows the PCA 3.7-6.25 keV light curve. The vertical lines represent the first radio detection in XTE J1748-288, and the first detection of optically thin synchrotron emission in XTE J1859+226.
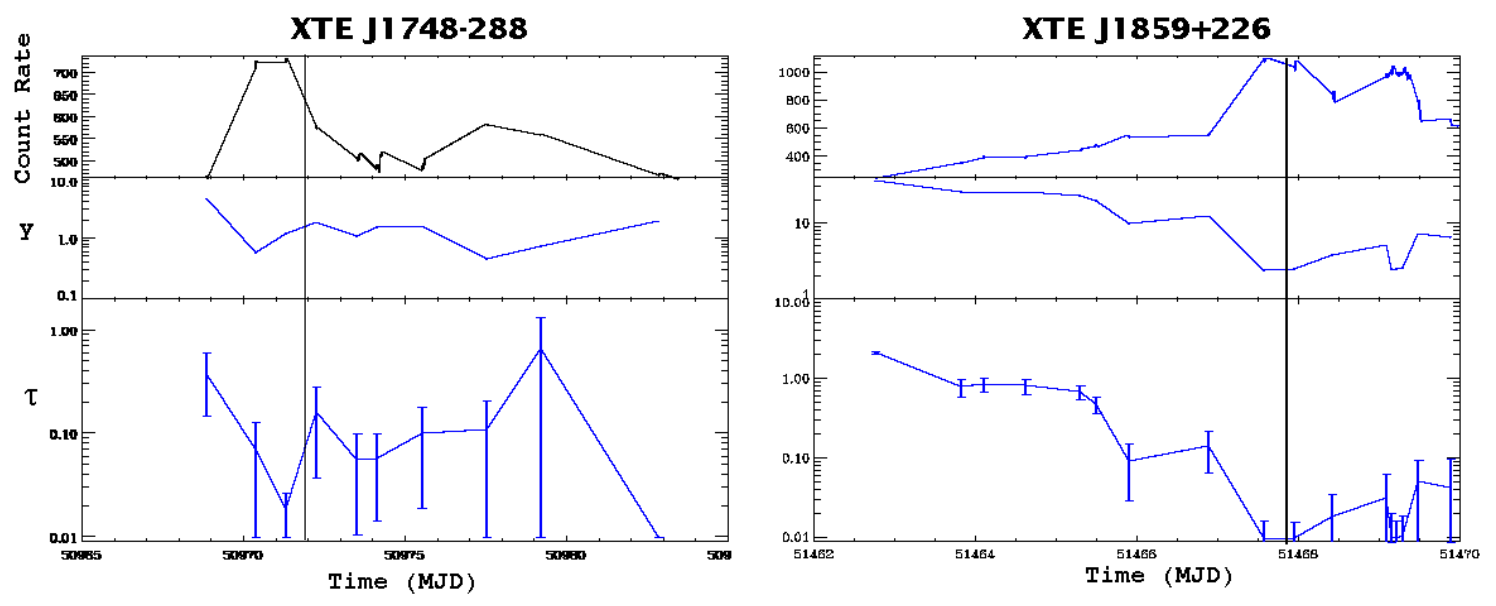

optical depth of the corona. $\max \left(\tau, \tau^{2}\right)$ is equivalent to the mean number of scattering, and one may further remark that $\tau \propto \rho \times R$, with $\rho$ the coronal density and $R$ the size of the corona. We represented the evolution of the $\tau$ parameters during the outbursts of XTE J1748-288 and XTE J1859+226 in Fig. 7. While the evolution of the fluxes does not indicate anything special concerning the corona, the evolution of the $y$ and $\tau$ parameters shows that something drastic occurred. These quantities indeed decrease by a factor $\geq 10$ in both sources. In each source it is remarkable that these drops occurred slightly before the radio ejections appeared. Recalling that $y$ is somehow related to the mean number of scattering, and that $\tau$ has the dimension of a surface density, these drops are therefore compatible with: a decrease of the number of coronal electrons (at constant coronal size), or a decrease of $R$ with a constant electron density, or even both effects combined. While the first effect could be due to sudden accretion of the corona, the latter two are equivalent to an ejection of the corona, since they would be equivalent to a net loss of mass. In any cases, the shrink of $\tau$ indicates that the corona sees its density decreasing, and that therefore it somehow looses matter. Note that, although we cannot rule out that a sudden accretion event has taken place, the detection of ejections soon after the disappearance of coronal medium again argues in favour of the ejection of part of the corona.

\section{Conclusions}

We have shown that in GRS 1915+105 and XTE J1550-564 the origin of the ejection could be the coronal material. The argument is based on the evolution of the fluxes of the different X-ray emitting media. In both cases, the flux of the corona is seen to decrease by a large factor before an ejection is detected in the radio domain. In order to test this simple model, we studied two other sources for which multiwavelength data are available. These sources, XTE J1748-288 and XTE J1859+226, showed standard outbursts, initiated by a hard X-ray flare, transition to a softer outburst, the peak of which preceded a radio flare indicative of a discrete ejection of matter. Contrary 
to GRS $1915+105$ and XTE J1550-564, the fluxes do not evolve in the same simple way in those two sources. In XTE J1859+226 we do not even see a drop in the flux of any of the two spectral components.

We remarked that the right parameters to properly characterise Comptonization is the $y$ parameter, and more precisely the optical depth of the corona $\tau$. When studying those two quantities, we saw that, for both sources, they dropped by a significant factor before the radio ejection was detected. Given that $\tau$ somehow relates to the corona density and radius, we interpret these observations as evidence that part of the corona is ejected. Of course more systematic multiwavelength monitoring of microquasars are necessary to further conclude and generalised the fact that the ejecta are composed of coronal material in all sources. Our approach showed, that although the details of their evolution were different, in all four microquasar the origin of the discrete ejection is tightly linked to the evolution of the properties of the corona. We interpret the coronal evolution, in all four sources, as a disappearance of part of this medium, that feeds the ejecta.

\section{Acknowledgements}

We acknowledge E. Kuulkers, R. Rothschild, M. del Santo, M. McCollough for useful interactions during the presentation of this work at the meeting. We particularly warmly thank M. Coriat, S. Corbel, and E. Koerding for constant stimulating exchanges and discussions, and a careful reading of this paper. This research has made use of data obtained through the High Energy Astrophysics Science Archive Center Online Service, provided by the NASA/Goddard Space Flight Center. It is also partly based on observations with INTEGRAL, an ESA mission with instruments and science data centre funded by ESA member states (especially the PI countries: Denmark, France, Germany, Italy, Switzerland, Spain), Czech Republic and Poland, and with the participation of Russia and the USA. The Green Bank Interferometer was operated by the National Radio Astronomy Observatory for the U.S. Naval Observatory and the Naval Research laboratory during the time period of these observations.

\section{References}

[1] Belloni, T., Klein-Wolt, M., Mendez, et al. 2000, A\&A, 355, 271

[2] Brocksopp, C., Fender, R.P., McCollough, M., et al. 2002, MNRAS, 331, 765

[3] Corbel, S., Fender, R. P., Tzioumis, A. K., Nowak, M., McIntyre, V., Durouchoux, P., Sood, R. 2000, A\&A, 359, 251

[4] Corbel, S., Kaaret, P., Jain, R.K., et al. 2001, ApJ, 554, 43

[5] Fender, R. P., Belloni, T. M., Gallo, E. 2004, MNRAS, 355, 1105

[6] Hjellming, R.M., Rupen, M.P., Mioduszewski A.J., 1998, IAUC 6934

[7] Homan, J. \& Belloni, T., 2005, Astrophys.Space Sci., 300, 107

[8] Klein-Wolt, M., Fender, R. P., Pooley, G. G., et al. 2002, MNRAS, 331, 745

[9] Mc Clintock, J.E. \& Remillard, R.A. 2006, Compact stellar X-ray sources. Eds Walter Lewin \& Michiel van der Klis. Cambridge University Press, 39, 157

[10] Markoff, S., Nowak, M., Corbel, S., Fender, R., Falcke, H. 2003, A\&A, 397, 645 
[11] Mirabel, I.F. \& Rodríguez, 1994, Nature, 371, 46

[12] Mirabel, I.F., Dhawan, V., Chaty, S., et al. 1998, A\&A, 330, L9

[13] Revnivtsev, M.G., Trudolyubov, S.P., Borozdin, K.N. 2000, MNRAS, 312, 151

[14] Rodriguez, J., Corbel, S., Tomsick, J.A. 2003, ApJ, 595, 1032

[15] Rodriguez, J., Corbel, S., Kalemci, E., Tomsick, J.A., Tagger, M. 2004, ApJ, 612, 1018

[16] Rodriguez, J., Hannikainen, D.C., Shaw, S.E., et al. 2008, ApJ, 675, 1436

[17] Rodriguez, J., Shaw, S.E., Hannikainen, D.C., et al. 2008, ApJ, 675, 1449

[18] Rybicki, G.B., \& Lightman, A.P., 1986 in Radiative Transfer in Astrophysics, Wiley-VCH

[Woods et al.] Wood, A., Smith, D.A., Marshall, F.E., Swank, J.E. 1999, IAUC Circ. 7274 